\title{
MECANISMOS DE CONTROLE PARA FLUXO DE VAPOR D'ÁGUA NA FLONA TAPAJÓS PARA O ANO DE 2002
}

\author{
Diego R. Aguiar ${ }^{1}$; Raimundo C. Oliveira Junior ${ }^{2}$; Raphael P. Tapajós ${ }^{1}$; Wilderclay M. \\ Bareto $^{1}$; Rodrigo da Silva ${ }^{1}$; Troy P. Beldine ${ }^{1}$ \\ ${ }^{1}$ Universidade Federal do Oeste do Pará - UFOPA \\ ${ }^{2}$ Empresa Brasileira de Pesquisa Agropecuária - Embrapa
}

\section{RESUMO}

O objetivo desse trabalho é analisar a relação do fluxo de vapor d'água com os mecanismos de controle (condutância de superfície, condutância aerodinâmica) para os períodos sazonais para o ano de 2002. Sítio de estudo está localizado na Floresta Nacional de Tapajós (FNT, $54^{\circ} 58^{\prime} \mathrm{W}, 2^{\circ} 51^{\prime}$ S, Pará, Brasil), próximo ao km 67 da Santarém-Cuiabá rodovia (BR-163). Na estação chuvosa a média de evapotrasnpiração (ETP) foi de 124,8 mm.mês-1, enquanto no seco obteve valor de 145,7 mm.mês-1. As médias para cada estação foram de 0,355 m.s-1 (chuvosa) e 0,206 m.s-1 (seca) para a condutância de superfície (Cs) e 0,325 m.s-1 (chuvosa) e 0,371 m.s-1 (seca) para condutância (Ca). Os mecanismos de controle reagem de formas diferentes durante os períodos sazonais para o fluxo de vapor d'água. Dessa forma, pode-se inferir que os mecanismos tem respostas de forma diferente para a evapotranspiração da floresta ao longo do ano.

\begin{abstract}
The aim of this work is to analyze the relationship of the flow of water vapor with the control mechanisms (surface conductance, aerodynamic conductance) for the seasonal periods for the year 2002. Study site is located in the Tapajós National Forest (TNF, $54^{\circ} 58$ 'N, $2^{\circ} 51^{\prime}$ S, Pará, Brazil), close to $67 \mathrm{~km}$ of the Cuiabá-Santarém highway (BR-163). In the rainy season the average evapotrasnpiração (ETP) was $124.8 \mathrm{~mm}$.mês-1, while in the dry obtained value $145.7 \mathrm{~mm}$.mês $\mathrm{s}^{-1}$. The averages for each station were $0.355 \mathrm{~ms}^{-1}$ (wet) and $0.206 \mathrm{~ms}^{-1}$ (dry) to the surface conductance (Cs) and $0.325 \mathrm{~ms}^{-1}$ (wet) and $0.371 \mathrm{~ms}^{-1}$ (dry) to conductance (Ca). Control mechanisms react differently during the seasonal periods for the flow of water vapor. Thus, it can be inferred that the mechanism has answers differently to evapotranspiration of the forest throughout the year.
\end{abstract}

\section{INTRODUÇÃO}

As florestas possuem alta evapotranspiração e maior rugosidade aerodinâmica em relação ao pasto e as culturas agrícolas elevam os níveis de umidade atmosférica e a convergência atmosférica, portanto, aumentam as probabilidades de formação de nuvens e, consequentemente, de chuva (PIELKE et al.,1998; BRUIJNZEEL, 2004).

A ciclagem hídrica em ecossistema tropical, a evaporação total, como a soma de evaporação do dossel úmido e seco, é um componente hidrológico de grande importância na determinação do balanço hídrico das áreas florestais devido aos volumes significativos envolvidos (MALHI et al., 2002; KUME et al., 2011).

Assim, o objetivo desse trabalho é analisar a relação do fluxo de vapor d'água com os mecanismos de controle (condutância de superfície, condutância aerodinâmica) para os períodos sazonais para o ano de 2002.

\section{MATERIAIS E MÉTODOS}


Sítio de estudo está localizado na Floresta Nacional de Tapajós (FNT, 54 $58^{\prime} \mathrm{W}, 2^{\circ}$ 51' S, Pará, Brasil), próximo ao km 67 da Santarém-Cuiabá rodovia (BR-163). O FNT é limitado pelo Rio Tapajós para o oeste e da BR-163 ao leste, estendendo-se desde $50 \mathrm{~km}$ a $150 \mathrm{~km}$ ao sul da cidade de Santarém, Brasil Pará. A torre está localizada $6 \mathrm{~km}$ ao oeste da rodovia BR-163 e 6 km a leste do Rio Tapajós.

Os dados foram coletados a partir de uma torre micrometeorológica equipada com o sistema Eddy Covarience, responsável por medir os fluxos de vapor e massa e também conta com anemômetros sônicos tridimensionais.

Modelo de Penman-Montheith (PM)

Para o calculo da evapotranspiração, foi utilizado o modelo de Penman-Montheith, por ser

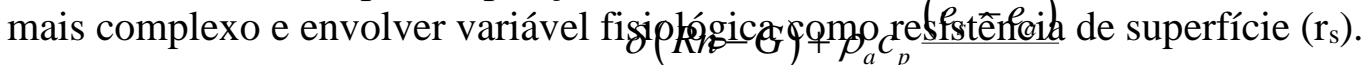

$$
E T P=\frac{r_{a}}{\delta+\gamma\left(1+\frac{r_{s}}{r_{a}}\right)}
$$

Equação 1

Em que:

ETP = Evapotranspiração diária $\left(\mathrm{mm}^{-1 a^{-1}}\right) ; \delta$ é a declividade da curva de pressão de saturação de vapor d'água $\left(\mathrm{kPa}{ }^{\circ} \mathrm{C}^{-1}\right) ; \mathrm{Rn}=$ saldo de radiação $\left(\mathrm{W} \cdot \mathrm{m}^{-2}\right) ; \mathrm{G}=$ fluxo de calor no solo $\left(\mathrm{W} \cdot \mathrm{m}^{-2}\right) ; \rho \mathrm{a}=$ densidade média do ar $\left(1,292 \mathrm{~kg} \cdot \mathrm{m}^{-3}\right) ; \mathrm{cp}=$ calor específico do ar à pressão constante $\left(\mathrm{J} \cdot \mathrm{kg}^{-1} \cdot \mathrm{C}^{-1}\right) ; \gamma=$ constante psicrométrica $\left(\mathrm{kPa} . \mathrm{C}^{-1}\right) ; \mathrm{rs}=$ resistência estomática $\left(\mathrm{s} . \mathrm{m}^{-1}\right) ; \mathrm{ra}=$ resistência aerodinâmica $\left(\mathrm{s} . \mathrm{m}^{-1}\right) ;$ ea $=$ pressão real de vapor $(\mathrm{Kpa})$; es = pressão de saturação de vapor $(\mathrm{Kpa})$.

Condutância de Superfície

$$
C_{s}=\left\{\frac{\rho_{a} c_{p} D P V}{\gamma L E}-r_{a}\left(1-\frac{\delta H}{\gamma L E}\right)\right\}^{-1}
$$

Equação 2

Sendo: Cs é a condutância de superfície $\left(\mathrm{m} . \mathrm{s}^{-1}\right)$; DPV o déficit de pressão de vapor $(\mathrm{kPa})$; $\rho$ a é a densidade do ar $\left(1,292 \mathrm{~kg} \cdot \mathrm{m}^{-3}\right)$; cp é o calor específico do ar úmido $\left(1,013 \mathrm{~J} \cdot \mathrm{Kg}^{-10} \mathrm{C}^{-1}\right) ; \gamma$ é a constante psicrométrica $\left(\mathrm{kPa}^{\circ} \mathrm{C}^{-1}\right)$;

Condutância Aerodinâmica

A condutância aerodinâmica $\left(\mathrm{C}_{\mathrm{a}}\right)$ é o inverso da resistência aerodinâmica $\left(\mathrm{r}_{\mathrm{a}}\right)$, (ALLEN et al., 1998). Para o cálculo da condutância aerodinâmica assumiu se a atmosfera não neutra, e será dada pela Equação 3 (CAMPBELL e NORMAN, 1998).

$$
\left(C_{a}^{-1}\right)=r_{a}=\frac{\ln \left[\frac{z_{m}-d}{z_{o m}}+\psi_{M}\right] \ln \left[\frac{z_{h}-d}{z_{o h}}+\psi_{H}\right]}{k^{2} U_{Z}}
$$

$\mathrm{zm}=$ medida da altura do vento $(\mathrm{m}) ; \mathrm{zh}=$ medição da altura da umidade $(\mathrm{m}) ; \mathrm{d}=$ altura do plano zero de deslocamento $(\mathrm{m})$; zom = comprimento da rugosidade que rege o momento da transferência $(\mathrm{m})$; zoh $=$ comprimento da rugosidade que rege transferência de calor e vapor $(\mathrm{m}) ; \mathrm{k}=$ von Karman's constant, $0.41 ; \mathrm{uz}=$ velocidade do vento na altura $\mathrm{z}\left(\mathrm{m} . \mathrm{s}^{-1}\right) ; \Psi_{\mathrm{M}}=$ Fator de correção para fluxo de momentum; $\Psi_{\mathrm{H}}=$ Fator de correção para fluxo de calor sensível.

\section{RESULTADOS}


O modelo PM obteve medias menores para o período chuvoso (124,8 mm.mês $\left.{ }^{-1}\right)$ e maiores para o seco $\left(145,7 \mathrm{~mm} . \mathrm{mês}^{-1}\right)$. Uma relação inversa ao regime de precipitação na região (Figura 1).

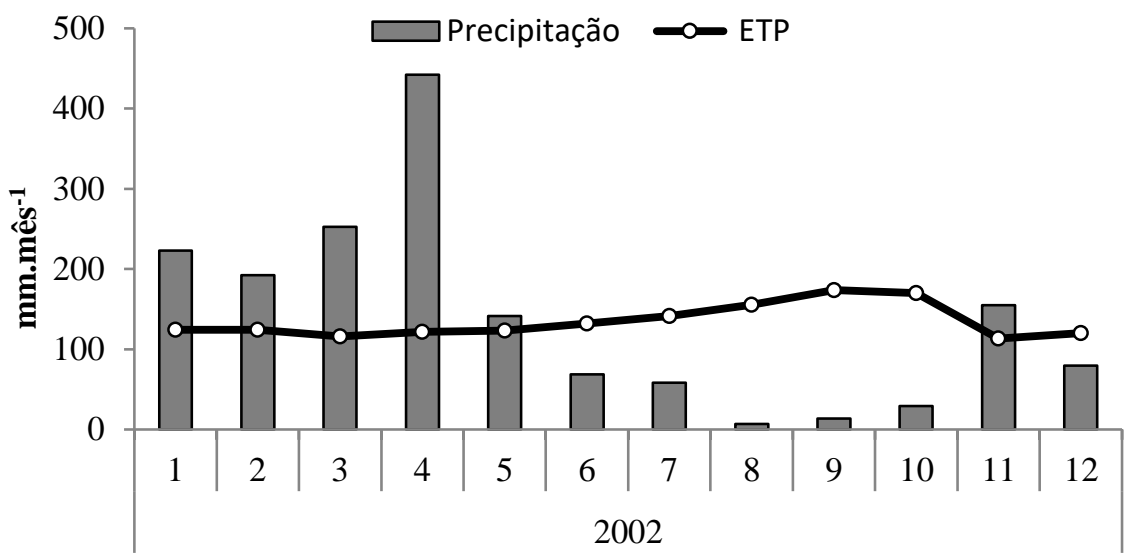

Figura 1 - ETP e precipitação para o ano de 2002.

Na estação chuvosa, a média de ETP foi de 124,8 mm.mês ${ }^{-1}$, enquanto na estação seca obteve-se o valor de 145,7 mm.mês ${ }^{-1}$. Essa variação de um período para o outro pode estar relacionada com as mudanças das variáveis atmosféricas sobre o dossel da floresta, que responde de maneira diferente para evapotranspiração de acordo com a estação do ano.

Para a condutância de superfície $\left(\mathrm{C}_{\mathrm{s}}\right)$ e aerodinâmica $\left(\mathrm{C}_{\mathrm{a}}\right)$, verificou-se uma tendência inversa. Enquanto a $\mathrm{C}_{\mathrm{s}}$ teve uma diminuição, a $\mathrm{C}_{\mathrm{a}}$ aumentou ao longo do ano de 2002 (Figura 2).

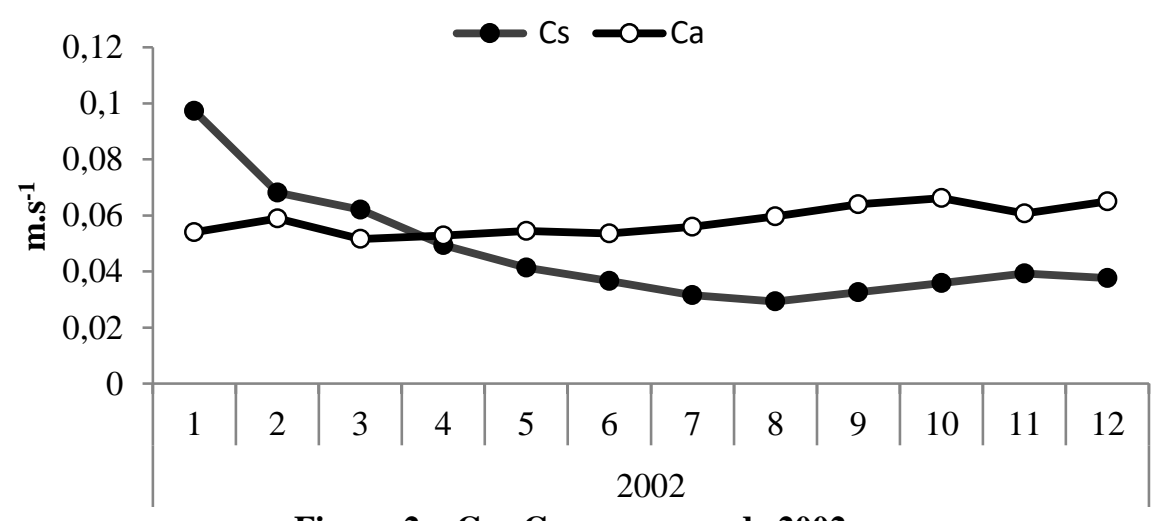

Figura 2 - $C_{s}$ e $C_{a}$ para o ano de 2002.

As médias para cada estação foram de $0,355 \mathrm{~m} \cdot \mathrm{s}^{-1}$ (chuvosa) e $0,206 \mathrm{~m} \cdot \mathrm{s}^{-1}$ (seca) para a Cs e $0,325 \mathrm{~m} \cdot \mathrm{s}^{-1}$ (chuvosa) e $0,371 \mathrm{~m} \cdot \mathrm{s}^{-1}$ (seca) para $C_{a}$. A variação sazonal da $C_{s}$ e $C_{a}$ na figura 2 mostra que estas variáveis têm sentidos opostos, pois quando uma tem valores decrescentes, outra possui uma crescente. Se for comparada a média de distribuição ao longo do ano, a $\mathrm{C}_{\mathrm{a}}$ tem variação semelhante a ETP.

$\mathrm{Na}$ tabela 1 abaixo, tem-se o acumulado para cada período, onde a precipitação e $\mathrm{C}_{\mathrm{s}}$ tiveram valores superiores para os primeiros seis meses (estação chuvosa). Já a ETP e $\mathrm{C}_{\mathrm{a}}$ obtiveram valores mais elevados para a estação seca. Dessa forma, as medidas de ETP e $\mathrm{C}_{\mathrm{a}}$ apresentam uma tendência semelhante para os períodos sazonais. Assim, a $\mathrm{C}_{a}$ é um dos mecanismos de fluxo de superfície que pode ter uma relação direta com o fluxo de vapor d'água para a atmosfera. 
Tabela 1 - Medidas sazonais de precipitação e ETP em mm.mês e condutância de superfície e aerodinâmica em $\mathrm{m} \cdot \mathrm{s}^{-1}$.

\begin{tabular}{lcccc}
\hline Estações & Precipitação & ETP & $\mathbf{C}_{\mathbf{s}}$ & $\mathbf{C}_{\mathbf{a}}$ \\
\hline Chuvosa & 1320,5 & 741,00 & 0,355 & 0,325 \\
Seca & 342,9 & 873,64 & 0,206 & 0,371 \\
\hline
\end{tabular}

Para melhor avaliar o quanto uma variável corresponde com a outra, abaixo na tabela 2 , tem-se a correlação entre as variáveis de estudos. As que possuem maior correlação são a ETP e $\mathrm{C}_{\mathrm{a}}$ com a precipitação.

Tabela 2 - Correlação das variáveis para o ano de estudo.

\begin{tabular}{lcccc} 
& ETP & Precipitação & $\mathbf{C}_{\mathbf{s}}$ & $\mathbf{C}_{\mathbf{a}}$ \\
\hline ETP & 1 & & & \\
Precipitação & $-0,65$ & 1 & & \\
$\mathbf{C}_{\mathbf{s}}$ & $-0,46$ & 0,56 & 1 & \\
$\mathbf{C}_{\mathbf{a}}$ & 0,56 & $-0,62$ & $-0,43$ & 1 \\
\hline
\end{tabular}

\section{DISCUSSÃO}

Segundo Vendrame et al., (2000), a medida de evapotranspiração tende a variar de acordo com o modelo. Sendo essa variável, foi calculada a partir de três modelos diferentes (Penman-Monteith, Maia Alves e Hydra) com valores de 3,6, 4,5 e 3,6 mm.dia ${ }^{-1}$, que em escala mensal de 108, 135 e 105 mm.mês ${ }^{-1}$, respectivamente na Reserva Jarú.

Diferentes fatores biofísicos controlam a transferência de vapor de água para a atmosfera. Dentre estes podemos destacar: a disponibilidade de energia; a demanda atmosférica por vapor de água; a condição aerodinâmica; e finalmente, por fatores fisiológicos da vegetação. Os valores obtidos de $\mathrm{C}_{\mathrm{s}}$ de $0,060 \mathrm{~m} \cdot \mathrm{s}^{-1}$ para a estação chuvosa e $0,045 \mathrm{~m} . \mathrm{s}^{-1}$ para a menos chuvosa (SOUZA FILHO et al., 2005).

$\mathrm{A} \mathrm{C}_{\mathrm{a}}$ no estudo de Rocha et al., (2004) no km 83 na Floresta Nacional do Tapajós, foi de $0,0287 \pm 0,0073 \mathrm{~m} \cdot \mathrm{s}^{-1}$ de julho de 2000 a junho de 2001, enquanto neste trabalho para o mesmo período obteve média de $0,071 \pm 0,01 \mathrm{~m} \cdot \mathrm{s}^{-1}$. De acordo com Rennó (2003) Monteith sugere um valor de $0,1 \mathrm{~m} . \mathrm{s}^{-1}$ para uma vegetação muito resistente como é o caso das florestas.

\section{CONCLUSÃO}

Os mecanismos de controle reagem de formas diferentes durante os períodos sazonais para o fluxo de vapor d'água. Levando em conta a correlação, os que possuem maiores valores foram o ETP e Ca com a precipitação. Dessa forma, pode-se inferir que os mecanismos têm respostas diferentes para a evapotranspiração da floresta ao longo do ano.

\section{AGRADECIMENTOS}


Agradeço ao Cnpq pelo apoio e fomento a pesquisa. Ao laboratório de processamento de dados Ambientais LBA - Santarém e ao grupo de pesquisa BRAMA.

\section{REFERÊNCIAS BIBLIOGRÁFICAS}

ALLEN, R. G. et al.. Crop Evapotranspiration, Food and Agriculture Organization of the United Nations, Rome, Italy. FAO publication 56, 1998, p. 290.

BRUIJNZEEL, L.A. Hydrological functions of tropical forests: not seeing the soil for the trees? Agriculture, Ecosystems and Environment, n. 104, p. 185-228, 2004.

CAMPBELL, G. S.; NORMAN, J. M. An introduction to environmental biophysics. New York: Springer-Verlag, 2nd ed., $286 \mathrm{p}$.

KUME, T., et al. Ten-year evapotranspiration estimates in a Bornean tropical rainforest. Agricultural and Forest Meteorology, n. 151, p. 1183- 1192, 2011.

MALHI, Y. et al. Energy band water dynamics of a central Amazonian rain forest. Journal of Geophysical Research, 107, p. 8061-8078, 2002.

PIELKE, R. A . et al.. Interactions between the atmosphere and terrestrial ecosystems:

influence on weather and climate. Global Change Biology, 4, p. 461-475. 1998.

RENNÓ, C. D. Construção de um sistema de análise e simulação hidrológica: aplicação a bacias hidrográficas. 2003. 158 p Tese (Doutorado em Sensoriamento Remoto) - Instituto Nacional de Pesquisas Espaciais, São José dos Campos.

ROCHA, E. J. P.. Balanço de umidade e influência de condições de contorno superficiais sobre a precipitação da Amazônia. 2004, f. 212. Tese de Doutorado em Meteorologia, Instituto nacional de pesquisas espaciais (INPE), São José dos Campos.

SOUZA FILHO, J. D. C. et al.. Mecanismos de controle da variação sazonal da transpiração de uma floresta tropical no nordeste da Amazônia. Revista: Acta Amazônica, vol. 35, p. 223-229, 2005.

VENDRAME, I.; ALBUQUERQUE, V. F.; FISCH, G. Influência da condutância estomática no cálculo da evapotranspiração em Rondônia. Revista Brasileira de Recursos Hídricos Volume 5, n. 2, p. 59-70, Abr/Jun 2000. 